\title{
Copper Sulfide - Zinc Sulfide Janus Nanoparticles and their Seebeck Characteristics for Sustainable Thermoelectric Materials
}

Hiroyuki Shimose', Maninder Singh'1, Dipali Ahuja', Wei Zhao², Shiyao Shan², Shunsuke Nishino ${ }^{1}$, Masanobu Miyata ${ }^{1}$, Koichi Higashimine ${ }^{1}$, Derrick Mott ${ }^{1 *}$, Mikio Koyano ${ }^{1}$, Jin Luo $^{2}$, C. J. Zhong ${ }^{2}$, Shinya Maenosono ${ }^{1}$

1 School of Materials Science, Japan Advanced Institute of Science and Technology, 1-1 Asahidai, Nomi, Ishikawa, 923-1292, Japan

2 Department of Chemistry, State University of New York at Binghamton, Binghamton, New York 13902, USA

XPS analysis of the sulfur $2 \mathrm{p}$ area for $\mathrm{Cu}_{2} \mathrm{~S}-\mathrm{ZnS}$ Janus Nanoparticles. Deconvolution of the sulfur $2 p$ peak was performed using a Gaussian function to fit $S 2 p_{3 / 2}$ and $S 2 p_{1 / 2}$ peaks for sulfide and thiolate species. The separation of $3 / 2$ and $1 / 2$ spin peaks was set to $1.18 \mathrm{eV}$ for both sulfide and thiolate while the peak intensity of the $1 / 2$ components was set to half the intensity of the $3 / 2$ spin peak, which is in accordance with literature values. The peak width for each species was allowed to be fit naturally (with the restraint that $3 / 2$ and $1 / 2$ spin peaks have the same peak width for each species), which resulted in the sulfide consistently having a narrower peak width (0.3-0.6 eV) while the thiolate consistently had a relatively broader peak width (0.8-1.1 $\mathrm{eV}$ ), which is consistent with literature reports. The full set of peak parameters is detailed below.

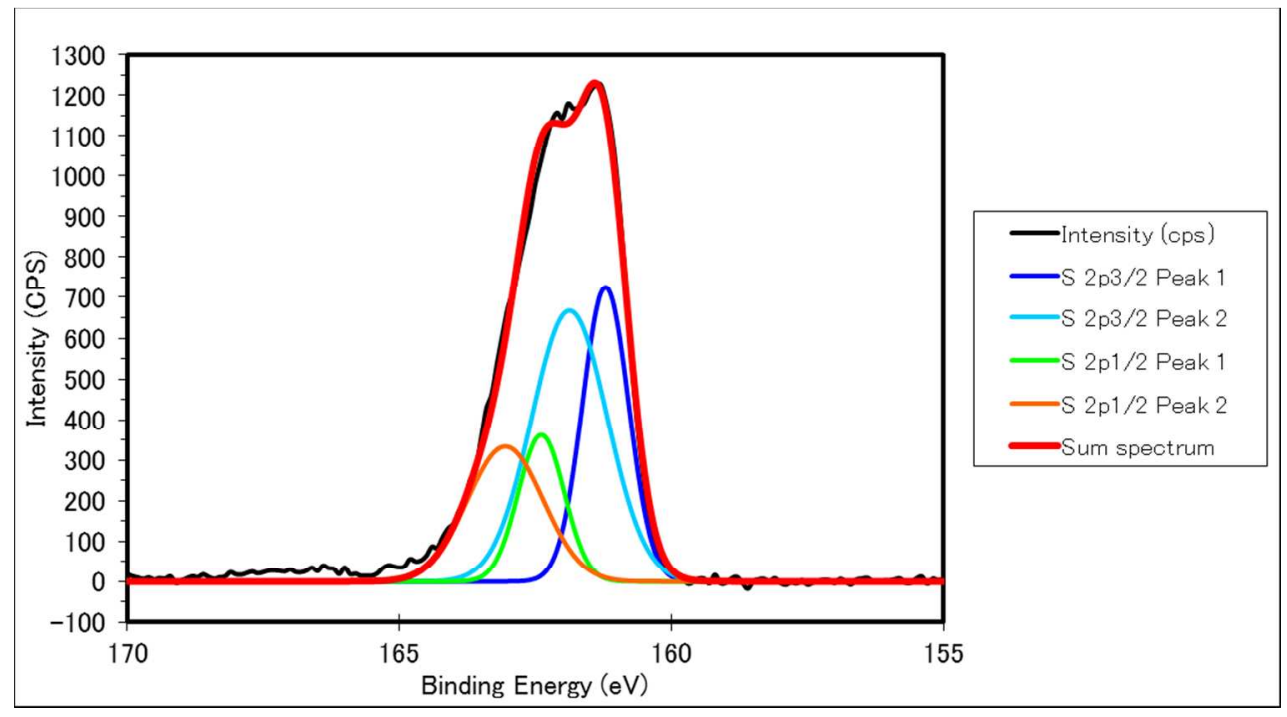

Figure S1. XPS spectra and deconvoluted components for $\mathrm{Cu}_{2} \mathrm{~S}$ nanoparticles (metallic feeding ratio of 4:0 $\mathrm{Cu}: \mathrm{Zn})$. 


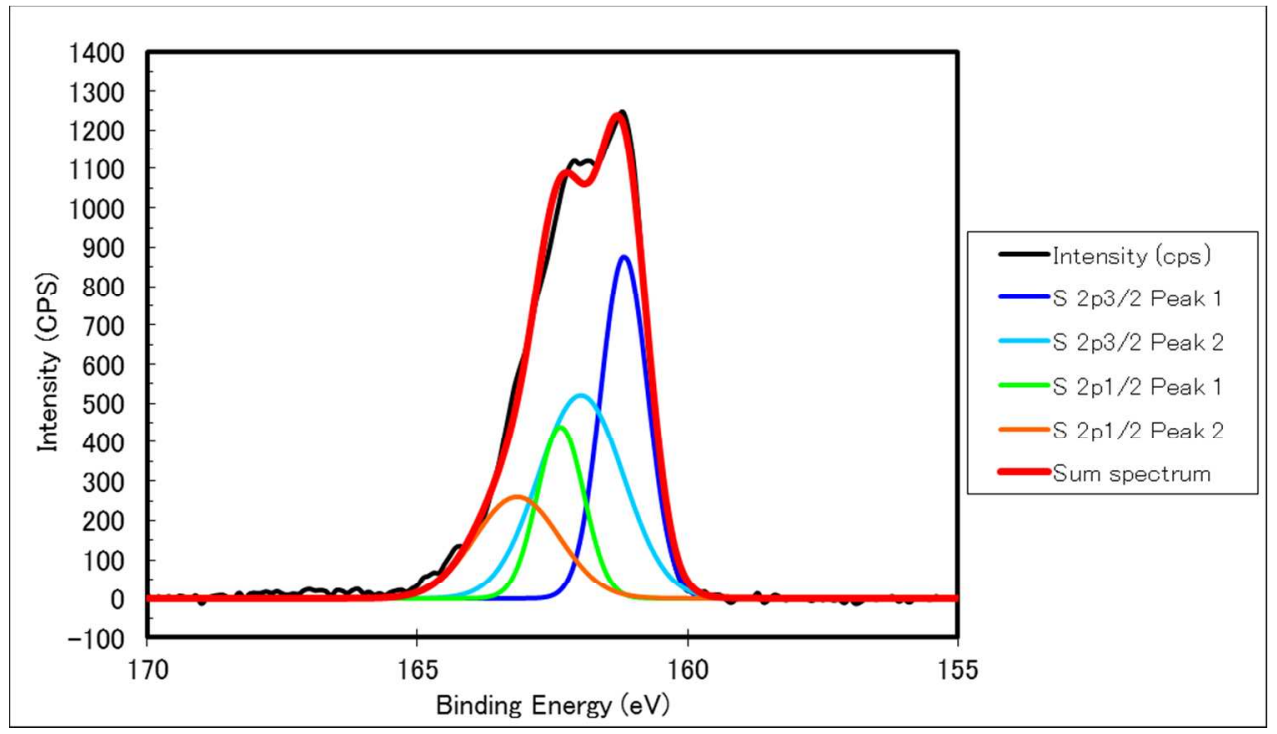

Figure S2. XPS spectra and deconvoluted components for $\mathrm{Cu}_{2} \mathrm{~S}-\mathrm{ZnS}$ nanoparticles (metallic feeding ratio of 3.5:0.5 $\mathrm{Cu}: \mathrm{Zn}$ ).

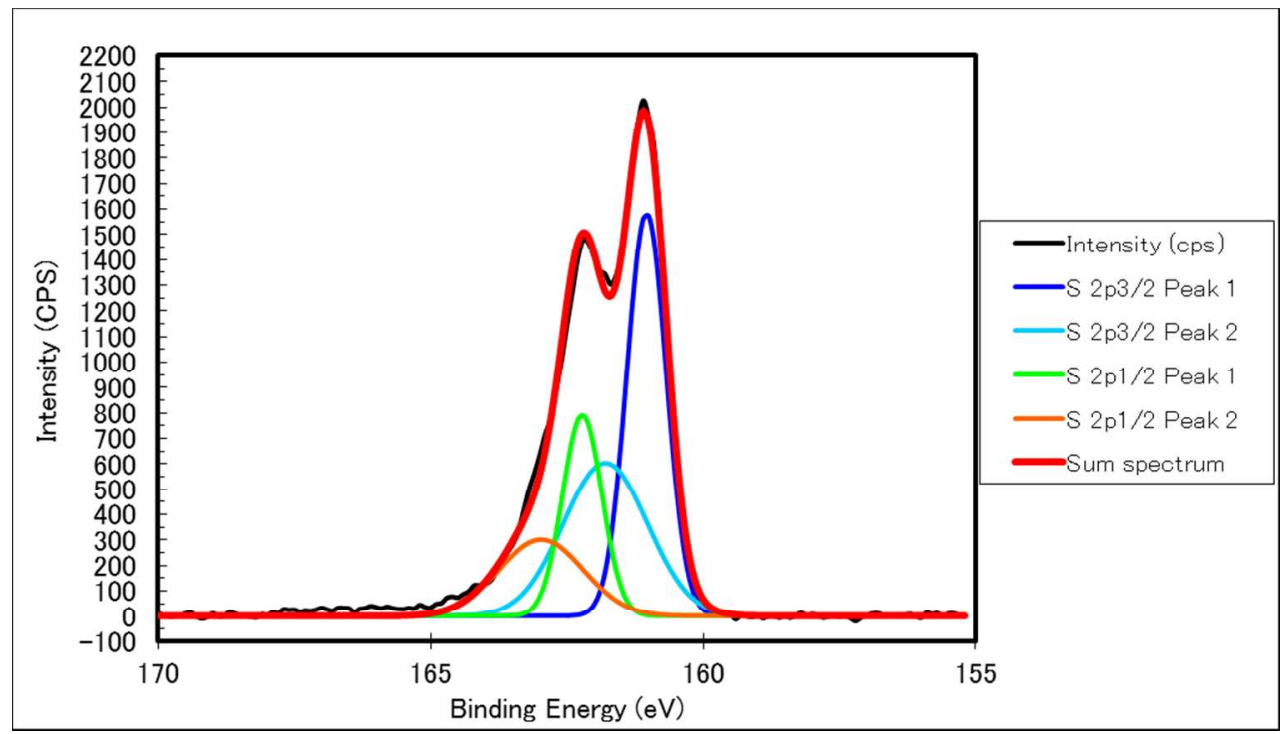

Figure S3. XPS spectra and deconvoluted components for $\mathrm{Cu}_{2} \mathrm{~S}-\mathrm{ZnS}$ nanoparticles (metallic feeding ratio of 3:1 Cu:Zn). 


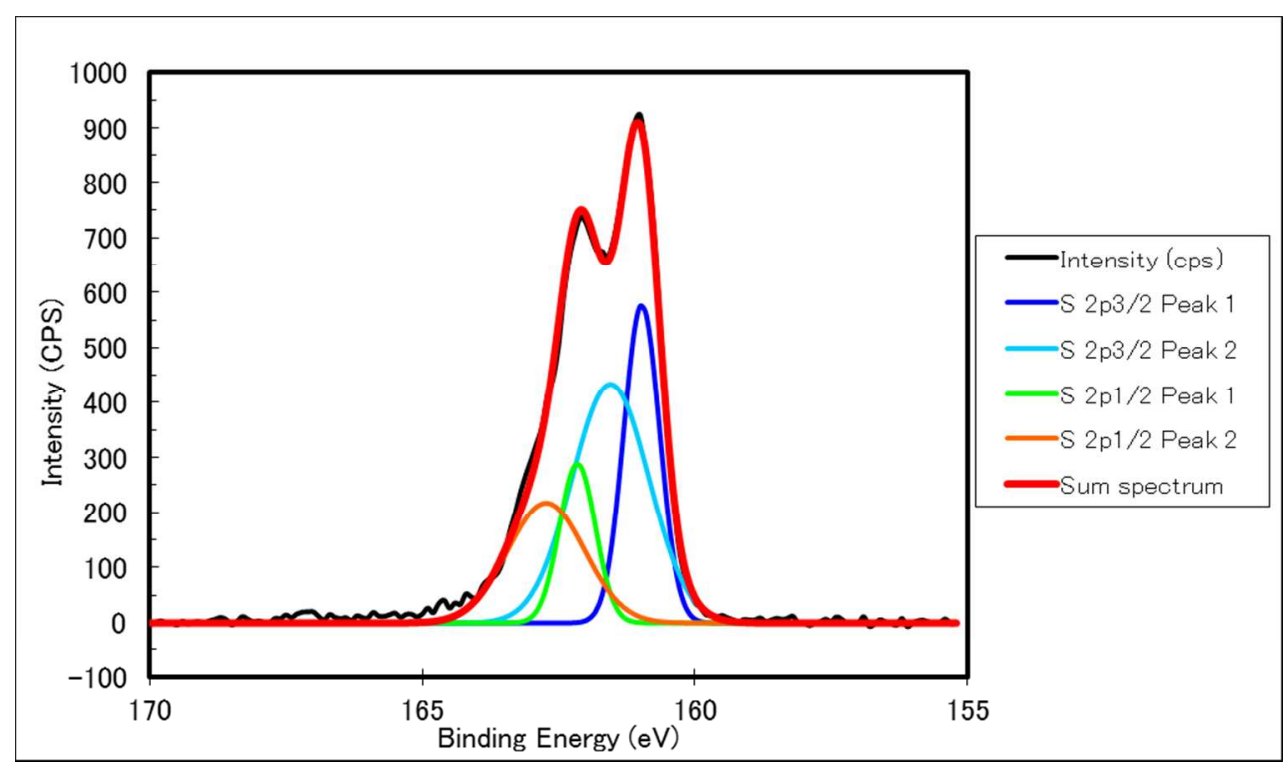

Figure S4. XPS spectra and deconvoluted components for $\mathrm{Cu}_{2} \mathrm{~S}-\mathrm{ZnS}$ nanoparticles (metallic feeding ratio of 2.5:1.5 Cu:Zn).

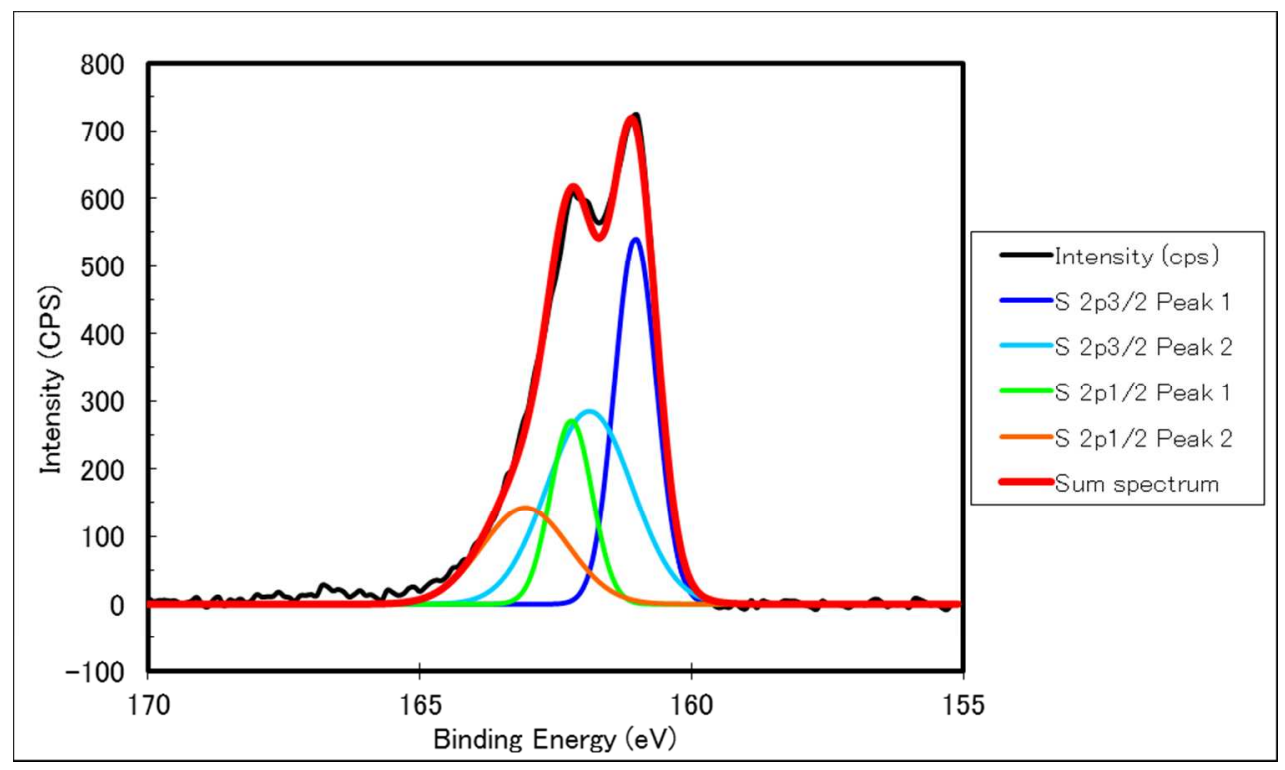

Figure S5. XPS spectra and deconvoluted components for $\mathrm{Cu}_{2} \mathrm{~S}-\mathrm{ZnS}$ nanoparticles (metallic feeding ratio of 2:2 Cu:Zn). 


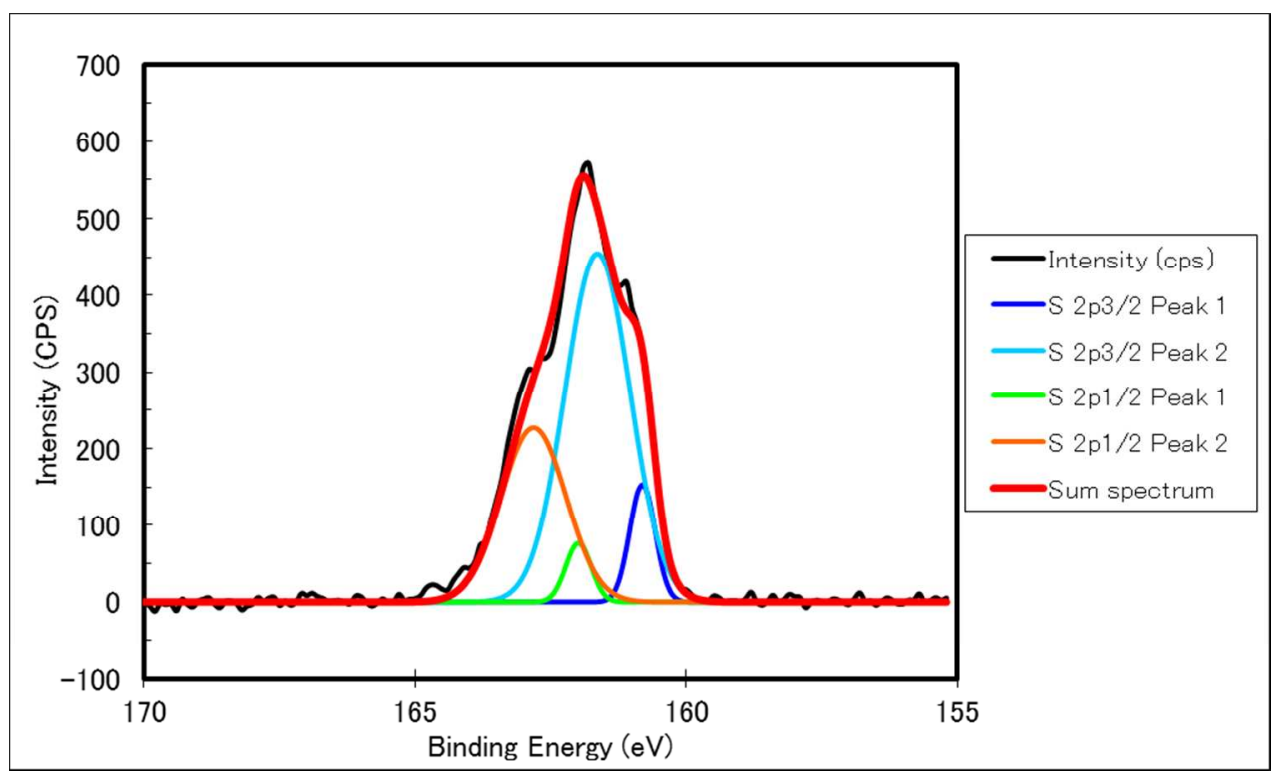

Figure S6. XPS spectra and deconvoluted components for $\mathrm{Cu}_{2} \mathrm{~S}-\mathrm{ZnS}$ nanoparticles (metallic feeding ratio of 1.5:2.5 Cu:Zn).

Table 1. Peak positions found by peak fitting (eV).

\begin{tabular}{|l|c|c|c|c|c|c|}
\hline & Cu4Zn0 & Cu3.5Zn0.5 & Cu3Zn1 & Cu2.5Zn1.5 & Cu2Zn2 & Cu1.5Zn2.5 \\
\hline S 2p $\mathbf{p}_{\mathbf{3} / \mathbf{2}}$ Peak 1 & 161.3 & 161.3 & 161.1 & 161.0 & 161.1 & 161.0 \\
\hline S 2 $p_{3 / 2}$ Peak 2 & 162.4 & 162.5 & 162.2 & 162.0 & 162.3 & 161.9 \\
\hline S 2 $p_{1 / 2}$ Peak 1 & 162.5 & 162.5 & 162.3 & 162.2 & 162.3 & 162.2 \\
\hline S 2 $p_{1 / 2}$ Peak 2 & 163.5 & 163.7 & 163.3 & 163.2 & 163.5 & 163.1 \\
\hline
\end{tabular}


Table 2. Peak intensity found by peak fitting (CPS).

\begin{tabular}{|l|c|c|c|c|c|c|}
\hline & Cu4Zn0 & Cu3.5Zn0.5 & Cu3Zn1 & Cu2.5Zn1.5 & Cu2Zn2 & Cu1.5Zn2.5 \\
\hline S 2 $\mathbf{p}_{3 / 2}$ Peak 1 & 1129.6 & 1161.7 & 1904.2 & 870.1 & 676.0 & 310.7 \\
\hline S 2 $\mathbf{p}_{3 / 2}$ Peak 2 & 382.5 & 336.6 & 544.1 & 316.0 & 240.5 & 403.2 \\
\hline S 2 $\mathbf{p}_{1 / 2}$ Peak 1 & 564.8 & 580.9 & 952.1 & 435.0 & 338.0 & 155.3 \\
\hline S 2 2 $\mathbf{p}_{1 / 2}$ Peak 2 & 191.3 & 168.3 & 272.0 & 158.0 & 120.3 & 201.6 \\
\hline
\end{tabular}

Table 3. Peak width found by peak fitting (eV).

\begin{tabular}{|l|c|c|c|c|c|c|}
\hline & Cu4Zn0 & Cu3.5Zn0.5 & Cu3Zn1 & Cu2.5Zn1.5 & Cu2Zn2 & Cu1.5Zn2.5 \\
\hline S 2 2 $\mathbf{p}_{3 / 2}$ Peak 1 & 0.6 & 0.6 & 0.5 & 0.5 & 0.5 & 0.3 \\
\hline S 2 $\mathbf{p}_{3 / 2}$ Peak 2 & 1 & 1.1 & 1.1 & 1 & 1.1 & 0.8 \\
\hline S 2 $\mathbf{p}_{1 / 2}$ Peak 1 & 0.6 & 0.6 & 0.5 & 0.5 & 0.5 & 0.3 \\
\hline S 2 2 $\mathbf{p}_{1 / 2}$ Peak 2 & 1 & 1.1 & 1.1 & 1 & 1.1 & 0.8 \\
\hline
\end{tabular}

